Annales Geophysicae (2001) 19: 1201-1205 C European Geophysical Society 2001

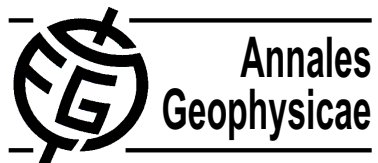

\title{
Topical tribune
}

\section{Space science and exploration in the $21^{\text {st }}$ century}

\author{
R. M. Bonnet \\ Director of Scientific Programme, European Space Agency, 8-10 rue Mario Nikis, F-75738 Paris Cedex 15, France
}

\section{Introduction}

The space era, initiated in 1957 with the launch of Sputnik-1, created in less than 50 years a genuine revolution in knowledge and our understanding of the Universe and of our own Solar System, which has no precedent in the history of the development of science. This is a clear illustration that the use of new technologies and techniques in astronomy has contributed to major scientific progress.

In parallel, the even faster development of electronic technologies and informatics, has also revolutionised the way we interact with space systems and the data they provide. This has profound implications of a sociological, ethical and philosophical nature. Through the use of work stations and the Internet, "big science" is brought to the level of individuals, literally "at home". Conversely, thousands of people (millions in the case of SETI) can work on the same problem and combine their individual contributions to develop a solution.

In addition, tremendous competition is seen between the space agencies and private institutions to bring scientific information to the public at large, even before the content of the data has been properly checked by the scientists. This situation was unthinkable even 10 years ago, and raises questions among the scientific community of a genuine ethical nature.

How can this activity continue and can we forecast its development in the near future? Can space telescopes continue to grow in size when the launchers of today have probably reached their maximum size? How many missions will we be able to launch to the various objects in the Solar System and, possibly outside the Solar System? How can we break the technological barriers which today make human exploration of the Solar System an unaffordable dream and outside the Solar System, impossible?

Is it, in fact, necessary to attempt to reach such a distant dream and if the answer is yes, how will we do it?

\section{Space astronomy for what?}

Without space, astronomy would only offer a very restricted and incomplete view of our Universe. Very little would be known about cosmic hot matter (only accessible through Xrays), cosmic nuclear matter (only accessible through gamma rays), cosmic cold matter and molecules (only accessible through infrared and submillimetric wavelengths). The ultimate challenge of astronomy is to observe the invisible and only space techniques through the unrestricted opening of the electromagnetic spectrum can assist us in reaching this goal. The total energy density of the Universe (Fig. 1) provides an illustration of its overall history and its evolution. In other words, to understand the Universe requires access to the whole electromagnetic spectrum. Therefore, space techniques are needed!

But is this sufficient? The answer is no! Light itself is not capable alone of revealing all the phenomena and structures in the Universe. For example, $90 \%$ of the mass of the Universe does not emit light: is it because we are not looking at the right part of the spectrum or because our telescopes are not sensitive enough, or both? Or is it also that this invisible mass is due to a new kind of matter or is a manifestation of a new physics? How can we access this hidden Universe? Probably thanks to a combination of efforts involving theorists, particle physicists and observers. We have no answer today!

Also the "Big Bang" is invisible because light was unable to escape the Universe during the first 300000 years of its existence. Only indirect methods allow us to conclude that it was a very probable event in the history of the Universe. However, other messengers of information have travelled unaltered from the "Big Bang" to us: the neutrinos and the gravitational waves. Neutrinos can be better detected from the ground (in fact, from underground). On the contrary, the gravitational waves which have been emitted during the "Big Bang" span frequencies in the range of $10^{-3}-10^{-1} \mathrm{~Hz}$ and can only be detected with space-borne detectors far away from the Earth where they are less sensitive to seismic perturbations. This is the objective of the LISA mission, a cornerstone of ESA's Space Science Programme and a joint project with NASA.

A word of caution is nevertheless necessary since it is not yet sure that these gravitational waves can be isolated from the added signals coming from a large variety of individ- 


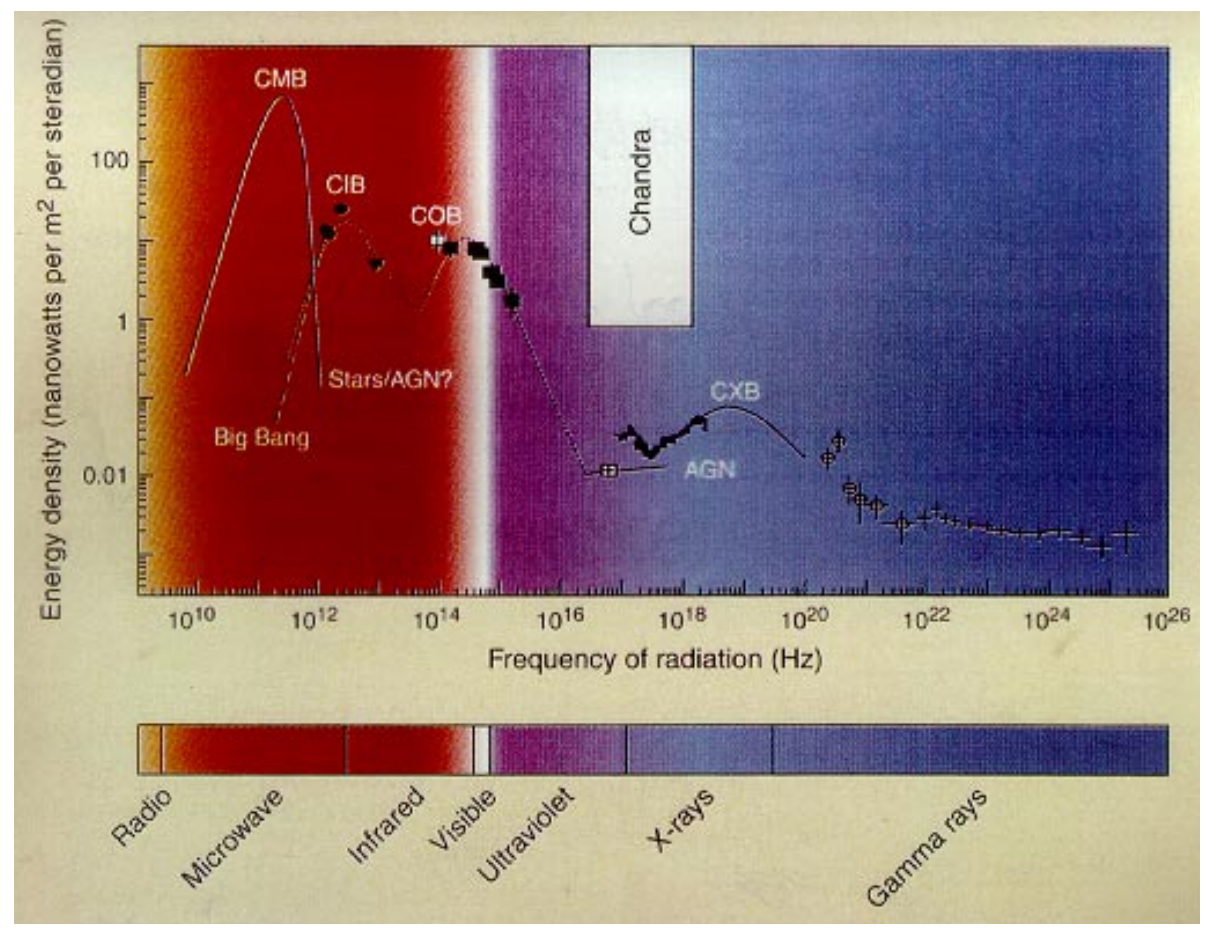

Fig. 1. Total energy density of the Universe.

ual sources. More theoretical work is, therefore, necessary. However, there is clearly hope that these waves may allow us to access events which have marked the very early Universe.

Black Holes are also structures which cannot be observed directly as their name indicates. Light is trapped in their enormous gravitational field. However, visible as well as Xray spectroscopy (matter is enormously heated while falling into a Black Hole) allow the measurement of the keplerian motion of matter in their vicinity. It has been confirmed that thanks, in particular, to the Hubble Space Telescope, every galaxy and quasar hosts a very massive Black Hole of several billion solar masses at their centre.

These examples illustrate, if necessary, that from a purely practical consideration, the future of astronomy is to develop more sensitive telescopes through new detectors and large mirrors over the totality of the electromagnetic spectrum.

Let us point out that telescopes do not yet exist for focussing gamma rays and the state of the art imaging device in this spectral range, i.e. ESA's Integral can only provide a few arc minutes resolution through the use of coded masks. Similarly, even the best X-ray telescopes cannot combine both angular resolution and photon collecting capabilities: the grazing incidence telescope of XMM-Newton is no larger than an equivalent visible telescope of $50 \mathrm{~cm}$ in diameter, even though it is still the largest ever produced!

In this context we see new concepts. ESA is studying the next generation of X-ray satellites which would couple high throughput and high angular resolution. The XEUS concept would be gradually assembled in space and would take advantage of the space station and make use of shell mirrors which have proved so efficient in the case of XMM-Newton.
In parallel, NASA is studying Constellation-X, a set of several individual satellites, each adding its capability to the others, thereby reaching a sensitivity 100 times larger than any previous individual telescope.

Even though visible astronomy is possible from the ground, HST has proven the tremendous power of observing above the Earth's atmosphere. Nevertheless, thinking bigger is always possible. For example, NASA's administrator has given NASA engineers a goal of developing a 50 to $100 \mathrm{~m}$ telescope. This is not realistic today, and NASA and ESA are now discussing the more modest NGST, which is scheduled to be launched in 2009. In fact, technology and cost reasons have forced the foreseen diameter of NGST to shrink already from 8 to 6 meters.

The fashion of today and most likely of tomorrow is the detection of extraterrestrial life and ultimately the observation of these planets which may harbour it. This pathetic quest has several justifications of a scientific as well as philosophical and religious nature, evidencing the basic and fundamental concern of humanity to understand its origins and possibly predict its fate. This theme also creates near unanimity in the expressed interest of the general public and has induced a large number of preparatory activities in the scientific community. We can mention, among others, small missions like COROT (CNES and ESA), Eddington (ESA) and Kepler (NASA) which are scheduled to be launched in this decade, with the primary goal of new planet detection.

However, the full development of this area will be witnessed with projects like Darwin in Europe and TPF in the US. Both missions rely on observations conducted in the infrared and both make use of interferometers. The near in- 
frared between 5 and $20 \mu \mathrm{m}$ hosts the most obvious spectral signatures of life, the bands of $\mathrm{O}_{2}, \mathrm{CO}_{2}, \mathrm{O}_{3}$ and $\mathrm{H}_{2} \mathrm{O}$, and it is ideal for the detection of extraterrestrial life.

Inferferometry will certainly mark the next century. It represents one of the best alternatives to the development of telescopes above $10 \mathrm{~m}$ in diameter. It would provide a solution to the present difficulties of launching larger single telescopes not only for the infrared but also for the visible and even for $\mathrm{X}$-rays.

The somewhat inconvenient drawback of decoding the unesthetic interferograms in order to obtain real pictures can be solved through software and the use of a larger number of telescopes. However, the recent experience acquired at ESA in operating the 4 identical Cluster satellites simultaneously tells us that handling an even larger number of satellites in a single mission will pose operational problems and raise new challenges.

From where should space astronomy be conducted in the future? Of course from above the Earth's atmosphere! We already mentioned XEUS which will use the space station. The Moon was also considered as a privileged observing site for large telescopes. This is unequivocally true for radio telescopes on the hidden side, but it is more debatable for all other spectral domains. In fact, today's tendency seems to go as far away as possible from the Earth, at Lagrangian point L2 or even further away, at distances of several astronomical units and even above the Ecliptic plane in order to eliminate the background of the zodiacal light.

At such places, the possibility of using astronauts to refurbish or to renew the hardware, as has been done on the Hubble Space Telescope, requires further thinking but is not impossible and NASA is studying it. Nevertheless, the large astronomical telescopes of the future may, for the time being and for a certain number of years, rely on "throw away systems"!

As seen here, the concept of smaller missions is not what the astronomers are dreaming of. In the context where budgets are capped, the competition with the rest of space science and, in particular, the exploration of the Universe in situ will continue to be fierce.

\section{Planetary exploration}

With the noticeable exception of Pluto and to a certain extent Mercury, all planets of the Solar System and some of their satellites have been observed from a close distance. Nevertheless, Mercury will soon be visited by of the NASA Messenger mission and of the ESA-ISAS BepiColombo mission. However, the fate of a mission to Pluto in NASA's programme is not yet ascertained.

Clearly apparent in the first part of this new century is the priority given to the exploration of Mars. The red planet is a privileged target for NASA who recently announced their plan to spend some 400 million US dollars annually in the future. The main space agencies in Europe, Russia and Japan have also declared war on Mars. Certainly the spectacular results of NASA's Mars Global Surveyor raise a lot of new questions about the past history of our sister planet.

Subjacent to this renewed interest is again the question of the existence of life in whatever form may have had time to evolve on the martian surface during the first billion years or so. The question of whether Mars ever saw running water still remains controversial. Even the very high resolution pictures of MGS have not yet been able to unambiguously solve the problem: where is the water, and does it continue to flow? Future US and European missions have some chance of resolving this enigma thanks to both radar-borne instruments and even higher resolution imagery.

The MARSIS radar on board the Mars Express is a must and a first. It will most certainly be followed by more performing instruments. Possibly a martian Synthetic Aperture Radar will hopefully provide a three-dimensional distribution of the underground water. From such observations, we should have a much improved insight into the Martian history of this crucial element in the development of life.

In spite of the negative results of the Viking missions, the search for life on Mars continues to agitate scientists and politicians. The dubious results of meteorite A184001 supposedly of martian origin has given more weight to in situ analysis of the red planet.

Here also, the Europeans have taken the initiative with the Beagle 2 lander on ESA's Mars Express mission. Beagle 2 will perform biochemical measurements from samples extracted from beneath the surface in regions which are the most likely to have kept the signature of life, i.e. in the shadow of boulders where the penetration of lethal solar UV radiation is shielded.

The geological history of Mars can be traced from the distribution of the minerals in the soil of the planet as provided by detailed infrared and X-ray spectroscopy. Such investigations will represent a large proportion of the payload capabilities of future missions. Landers such as Beagle 2, the Netlander mission of CNES and the future US missions will complete the picture by providing the most accurate measurements in pre-identified areas of interest.

These examples raise, at this point, the question of the urgency of the Mars sample return missions. The great complexity and the cost of such missions lends more credibility to the approach of performing, in priority, in situ measurements which, in addition, run less risk of biological contamination.

On both the Rosetta mission to Comet Wirtanen and BepiColombo, which will land on Mercury, ESA has studied the possibility of returning samples but has given priority to in situ analysis. The study also by ESA of a Venus Sample return scenario has demonstrated a fortiori the fairly obvious unaffordability of such a mission. Hence, it is not too surprising that sample return missions should follow and not precede in situ analysis.

Beyond Mars, the next few years will also, to a large extent, be driven by the search for water and for prebiotic activity in other parts of the Solar System. If, as is likely, the present problems which affect the transmission of data from Huygens to Cassini can be solved, and if the payload of Huy- 
gens performs nominally, we should learn a lot about the mysterious Titan, and I would hope that the unique conditions existing on that moon of Saturn, which concern prebiotic chemistry in the Solar System, will show up from the various instruments of its payload, and, in particular, of its surface science package.

Hence, if the success of Huygens is confirmed, we may, in the not too distant future after 2004, see a new mission to Titan with more elaborate instruments and possibly a longer stay on the surface. NASA has already identified such a mission in its strategic plan for the Titan Explorer and the problem for Europe will be to decide how it could exploit its visionary initiative of 1992 to go and land thereon for the first time.

NASA again is planning missions to the icy moons of Jupiter: Europa and Ganymède. The existence of a subsurface ocean on Europa, if confirmed, may offer another exciting opportunity to unravel the possible existence of prebiotic conditions in other worlds.

The study of the Sun itself after SOHO, has regained a large interest. NASA has initiated the "Living with a Star" programme, while ESA has selected the Solar Orbiter mission to fly above the ecliptic plane in the time frame of 2010 2013, and Japan is already building Solar-B. The three agencies together with Russia are discussing the possibility of coordinating these missions in the frame of the Inter Agency Consultative Groups under what could be the "International Living with a Star" programme. The relevance of this programme with the study of the Sun-Earth connection is one of its most important scientific and political asset. However, one of the first elements to be implemented, the Solar Probe, has been the victim of the new US administration and will have to wait until better times to be approved.

But will Europe itself be able to follow such initiatives while maintaining its own programmes? It is certainly more a matter of finances than one of competencies.

\section{The international context}

With respect to the amount of money spent in the world for space science, the dominating role of the US is obvious. The problem which Europe faces here is probably not of a scientific nature but of a political nature.

Can and will Europe be recognised in future years for its role as a source of ideas and contributions of advanced technologies and discoveries? Will it be able to maintain its creative forces for the benefit of the overall European society(ies)? Probably not, if the present level of support of the ESA Member States remains as it is, even in the context where the new US administration re-orientates its priorities, giving more importance to the military than to the civilian space effort.

In fact, the capacity of the US government and its industrial work force to transfer priorities from the civilian to the military space effort and vice versa is the very sign and the deep-rooted reason of the yet unbeatable leadership role of the US. We can summarise the situation as follows. The US is a nation with a vision and they have a mission to defend and protect themselves. This is the reason why they are a leader!

As for Europe? It consists of some 20 nations, each one with its own vision, its own education system, and no common defence. Nevertheless, in space, Europe has been able, up to now, to maintain a number two position, reaching even the first rank in some fields such as launchers, space astrometry, cometary physics, infrared astronomy, and helisopheric Physics. This was possible primarily because the US had not made the right decision (Halley's mission) or had not realised the value of the idea (astrometry), or had not selected the right concept (infrared astronomy) at the right time. It was certainly not a matter of technical incompetency.

Who are the other challengers? Besides Europe, Russia has not followed the visionary programmes of the Soviet Union and it is not clear when the immense scientific and intellectual capacities of that country will resurrect and interfere constructively again. Japan, like Europe, is stagnating. Next is China who is fairly far behind, indeed, but for how long? China's tremendous work force and intellectual capability and its continuous rise in the conquest of space through, in particular, the staged implementation of its future manned programme may indeed give it a leading position! If China continues, on this same part, Europe's ranking position of number two may well be challenged in a decade!

Today, maintaining Europe in that position is a decision of great political significance. The recent endorsement by both the EU Council and the ESA Council last December of a common European Space Policy is a very important and positive sign that the importance of space for the European societies and their industries is perceived in the political circles.

In the present budgetary context of Europe, and in view of the severe agricultural problems it is now facing, one should not place too much hope in a drastic reversal of the financial situation of the space programme in the very near future. Nevertheless, when the ESA ministers will meet next November in Edinburgh, they probably will have to decide on new programmes, among which is a preparatory programme for Planetary Exploration. The recent call for mission ideas for Planetary Exploration in order to prepare for that decision should be seen as a sign of interest by the governing bodies of ESA, and a manifestation of their willingness not to let the entire field of Planetary Exploration land in the hands of the US alone.

\section{Preparing the future}

In fact, the US is embracing the $21^{\text {st }}$ Century as if they wished to make their present leadership role unbeatable! Who could complain? Certainly not the space scientists who will be offered good opportunities to carry their observations or their instruments in the right orbit or on the right object. Nevertheless, should not Europe also prepare for the future? 
It is unlikely that a European defence will soon emerge at least at the same level of funding as in the US. Is it, in fact, absolutely necessary? No, if an alternative is offered in technological developments which space programmes, especially the space science programmes, can efficiently induce, thus maintaining a high technical, engineering, and scientific level of European citizens.

Larger space telescopes, which performing more planetary missions and robots as future infrastructures to be developed on the Moon, on Mars and perhaps on Titan, all rest on very advanced technologies. All of them are firmly in the hands of Europeans scientists and engineers, providing, of course, that they are granted the right level of financial resources and that they have not all fled to the US. At this stage, no idea should be eliminated a priori, such as, for example, launching human beings to the red planet. In fact, this may look to some as less crazy than building a large European military effort.

Landing on Mars requires, indeed, revolutionary technological developments, such as advanced propulsion, including nuclear propulsion, energy sources and advanced robotics in view of setting the proper infrastructure which will allow future generations to live on Mars, communicate with us and fly back to Earth at an affordable price. Anyone of these developments would benefit the overall space science effort and should be looked at positively.

However, no foreseeable extrapolation of existing technologies exist that would make the exploration of other planetary systems in our nearby neighbourhood look possible. Such possibilities do really transcend even the present achievements of science. Therefore, the exportation of life from Earth may, for a long time, be confined to our own So- lar System, and most likely to Mars. Nevertheless, I see no reason why Europe should not also fully take part in this exercise.

\section{Conclusion}

Indeed space science and planetary exploration will continue and expand, and probably will adopt even more ambitious aims in this century. Unless something is done to embrace this challenge or at least to prepare for it, such programmes will most likely be in the hands of the US alone. Certainly Europe can contribute a lot, both technically and scientifically to these programmes in the framework of collaborative ventures. However, its voice in present or future negotiations will be choked more and more if it does not represent a substantial and credible work force.

For that, it is not only necessary to invest more government money in space science programmes, but it is also crucial that the scientific and technical work forces should not emigrate to the US where they will be undoubtedly and cleverly utilised to re-inforce the US leadership, thereby amplifying the difference and the potential marginalisation of Europe. European brains will be more easily drained across the ocean when careers and salaries in Europe are not as attractive as compared to those in the US.

The winners of the $21^{\text {st }}$ Century are those who will invest enough to create and exploit education and knowledge for their benefit. The US is on the winning track; Europe has still some way to go! But it can also win, providing a strong political vision prevails at the level of the Union. 